\title{
Il campione per esame batteriologico e micobatteriologico: appropiatezza pre-analitica
}

\author{
Anna Calì', Giorgio Mucignat ${ }^{2}$, Rossella Sartori $^{3}$ \\ ' Microbiologa, delegata AMCLI regione Trentino Alto Adige \\ ${ }^{2}$ U.O. Microbiologia Clinica e T.A.A.O. di Pordenone \\ ${ }^{3}$ Dirigente Biologo Laboratorio Microbiologia e Immunologia Ospedale S. Chiara Trento
}

\section{INTRODUZIONE}

Gli esiti degli esami batteriologici e micobatteriologici sono fortemente condizionati dalla qualità del campione. L'appropriatezza della fase preanalitica implica una conveniente selezione, un'appropriata raccolta e un tempestivo trasporto di tutti i campioni.

Un campione non idoneo può determinare il mancato isolamento dei microrganismi veri responsabili dell'infezione e il riscontro di batteri contaminanti o del normale microbiota; in un certo senso chi raccoglie $i$ vari campioni tiene nelle sue mani il percorso diagnostico del paziente.

\section{Quali regole generali deve rispettare chi racco-} glie il campione?

- La raccolta del campione deve avvenire prima della terapia antibatterica e nei periodi ottimali per lo stadio dell'infezione.

- La raccolta del campione deve essere effettuata nella sede anatomica dell'infezione.

- Prelevare una quantità sufficiente di materiale.

- Evitare ogni contaminazione esogena ed endogena.

- Utilizzare appropriati sistemi di raccolta. (l'uso di mezzi sterili e una tecnica asettica per raccogliere i campioni previene l'introduzione di microrganismi soprattutto durante le manovre invasive)

- Contrassegnare il contenitore del campione con il nome del paziente, il numero di identificazione, la data e l'ora del prelievo.

- Identificare correttamente la natura e l'origine del campione affinché possano essere selezionati i più idonei terreni di coltura e le tecniche diagnostiche.

- Consegnare prontamente i campioni al laboratorio(per garantire la sopravvivenza e l'isolamento dei microrganismi fastidiosi, prevenire la sovracrescita dei batteri più resistenti, per ridurre il tempo di contatto del campione con alcuni anestetici locali usati nella procedura di prelievo con possibile azione antibatterica).

- Adottare, quando possibile idonee alternative alla consegna immediata.

- Fornire adeguate notizie cliniche al laboratorio.
- Per i prelievi particolarmente invasivi considerare il rapporto rischio/beneficio per il paziente.

\section{Cosa deve fare il laboratorio?}

- Definire precise linee guida sulle modalità di prelievo, conservazione e trasporto, specifiche per ogni tipo di campione.

- Monitorare la loro osservanza e applicazione.

- Predisporre percorsi diagnostici concordati con i clinici, perché sia chiaro quando si richiedono esami non abituali.

\section{I० PARTE}

\section{Anna Maria Calì}

\section{IL CAMPIONE FECALE}

Accettare un campione di feci raccolto e conservato impropriamente può determinare il riscontro di una coltura falso negativa, con conseguenze non solo per il singolo paziente, ma anche per la comunità per il rischio di non saper identificare outbreak epidemici di infezioni gastroenteriche.

Un ente americano per la verifica di qualità nei processi diagnostici sottopone un certo numero di laboratori al seguente quesito: la scena descrive un tecnico di laboratorio biomedico che, controllando il frigorifero dell'area dell'accettazione, trova un campione di feci vecchio di 3 giorni, raccolto da un paziente esterno in un vaso di burro di arachide e conservato in frigorifero per un tempo indeterminato.

La domanda era cosa dovrebbe fare il laboratorio con il campione? Noi cosa rispondiamo e perché?

\section{Step essenziali per il prelievo, conservazione e trasporto delle feci}

\section{Quando raccogliere il campione?}

- Il materiale deve essere raccolto nel momento acuto del processo infettivo, nel periodo di diarrea attiva, comunque il più presto possibile dopo l'insorgere dei sintomi, preferibilmente entro 4 giorni.

- Prima del trattamento antibiotico.

\section{Quanto e quale materiale raccogliere?}


- Per le colture di routine sono sufficienti 1-2 gr di feci, l'equivalente di una nocciola.

- In caso di feci diarroiche 1-2 ml, l'equivalente di un cucchiaino pieno.

- Se presenti scegliere le parti contenenti muco, pus o sangue.

- Il campione fecale non deve essere contaminato da urine.

\section{Quanti campioni raccogliere?}

Anche se un singolo campione di feci adeguatamente raccolto e conservato è sufficiente per fare diagnosi, nella maggior parte dei casi di enterocolite e alcuni studi, soprattutto in età pediatrica, confermano tale evidenza, si ricorda che per aumentare la possibilità di isolamento è opportuno esaminare 3 campioni raccolti e/o prelevati in giorni diversi.

Processare solo un campione al giorno dello stesso paziente; in caso di campioni multipli considerarli come unico prelievo.

L'esame colturale standard, per patogeni enterici acquisiti in comunità, non va eseguito su pazienti ospedalizzati da $>$ di 3 gg e senza una diagnosi di ammissione per enterocolite.

\section{Come raccogliere il campione?}

Le linee guida per la raccolta dei campioni fecali indicano 2 modalità:

- Feci emesse spontaneamente, campioni utili per l'esame colturale standard di Salmonella, Shigella, Campylobacter, ma anche di Yersinia, Vibrio, Aeromonas, E. coli enteropatogeni, per la coltura e la ricerca di tossine di Clostridium difficile, ricerca antigene di Helicobacter pylori e quando clinicamente indicato per la ricerca di Micobatteri.

Il paziente deve evacuare in un recipiente pulito ed asciutto senza residui di detergenti o disinfettanti; per raccogliere le feci non usare carta igienica, potrebbe essere impregnata di sali di bario, inibitori per alcuni patogeni enterici. Utilizzare l'apposito cucchiaio o la paletta in polipropilene fissata al tappo del contenitore fornito dal laboratorio. prelevare una adeguata quantità di materiale fecale, trasferirla nel contenitore e riavvitare il tappo accuratamente.

- Tamponi rettali.

L'uso del tampone rettale deve essere limitato a quei pazienti per i quali è obbiettivamente difficile la raccolta del campione fecale, bambini molto piccoli o pazienti non collaboranti; è indicato inoltre per la ricerca in gravidanza di portatrici di Streptococco betaemolitico di gruppo B. Il tampone rettale non è un campione accettabile per la ricerca delle tossine di $C$. difficile, per la ricerca di $\mathrm{Ag}$ di $H$. pylori e dei Micobatteri.
Pulire la zona anale con acqua calda senza usare soluzioni disinfettanti; inserire il tampone nel canale rettale per circa $2 \mathrm{~cm}$; lasciare inserito il tampone per circa 30", ruotandolo contro le pareti delle mucose. Verificare che sia visibile materiale fecale sul tampone.

\section{Modalità di conservazione e trasporto del campione di feci}

- Consegna immediata al laboratorio e processazione entro $2 \mathrm{~h}$.

Le attuali linee guida e i testi di riferimento raccomandano di processare i campioni di feci senza conservante entro $2 \mathrm{~h}$ dalla raccolta per mantenere l'integrità del campione. Alcuni enteropatogeni importanti come le Shigelle ma anche Vibrio e Campylobacter possono non sopravvivere agli effetti tossici e ai cambiamenti di $\mathrm{pH}$ che si verificano per la sovracrescita della normale flora batterica, anche se i campioni vengono refrigerati.

- Alternative alla consegna immediata: se il campione non può essere processato entro 2 ore dalla raccolta va mescolato ad un terreno di trasporto, quali Cary-Blair o soluzione glicerosalina tamponata; quest'ultima è fortemente raccomandata per la Salmonella e la Shigella, ma non per Campylobacter e Vibrio, a meno che non sia arricchita con CaCL2 (100 mg/l). Il Cary-Blair è raccomandato come un buono e versatile terreno di trasporto, sebbene non sia così adatto come la soluzione glicero salina tamponata per la sopravvivenza della Shigella. Per il trasferimento del campione che non può essere processato entro $2 \mathrm{~h}$, utilizzare un tampone sterile per prelevare una piccola quantità di feci, in particolare raccogliendo muco o frammenti di epitelio intestinale, quando presenti, e inserirlo immediatamente nel terreno di trasporto.

I campioni con conservante dovrebbero essere refrigerati fino al momento della semina; se devono essere conservati per $>$ di 3 giorni è preferibile il congelamento immediato a $-20^{\circ} \mathrm{C}$. Infatti è possibile effettuare la ricerca dei patogeni enterici da campioni congelati fino a $7 \mathrm{gg}$ dalla raccolta.

Non utilizzare terreno di trasporto per i campioni destinati alla ricerca di $C$. difficile, di $\mathrm{Ag}$ di H. pylori e Micobatteri

\section{Altri campioni dall'apparato gastroenterico per ricerca particolari:}

- Tampone anale: si utilizza per la ricerca di Gonococco, Chlamydia ed Herpes virus in caso di infezioni a trasmissione sessuale.

Introdurre il tampone nell'orifizio anale e ruotare per campionare le cripte anali e successiva- 
mente introdurlo negli appositi sistemi di trasporto.

- Lavaggio gastrico: viene utilizzato, anche se di rado, per la ricerca di Micobatteri in pazienti, più frequentemente bambini, incapaci di espettorare. Deve essere raccolto preferibilmente a digiuno e al risveglio in modo che sia raccolto materiale ingoiato durante la notte. È comunque da ricordare che sia le feci che l'aspirato gastrico sono campioni di scarso valore diagnostico per l'esame microscopico a causa della presenza di B.A.A.R. ambientali e saprofitici.

- Biopsia gastrica utilizzata per la coltura di $H$. pylori e Micobatteri.

- Biopsia intestinale utilizzata per la diagnosi di tubercolosi intestinale.

7. Ricapitolando, quali sono allora i criteri specifici di non accettabilità di un campione di feci?

- Tutti i campioni di feci formate, eccetto quando richiesta la ricerca di $S$. typhi, o indagini di Sanità pubblica per individuare portatori sani.

- Campioni di feci senza terreno di trasporto prelevati da $>$ di $2 \mathrm{~h}$.

- Campioni in terreno di trasporto con l'indicatore virato al giallo, che evidenzia il fallimento del sistema tampone per mantenere il $\mathrm{pH}$ neutro.

- Tamponi rettali secchi.

- Campioni multipli nello stesso giorno.

- Campioni da pz ospedalizzati da > di 3 gg per esame colturale standard.

- Se si riceve un campione non accettabile, notificare il problema al clinico e documentare sui piani di lavoro.

\section{Il campione urine}

La coltura quantitativa del campione di urine è fondamentalmente critica per porre diagnosi di infezione del tratto urinario. I criteri usati per distinguere tra crescita significativa e no dipendono molto dal tipo di campione ricevuto e processato. I campioni possono essere divisi in 3 categorie in base ai criteri clinici, alle possibilità di contaminazione uretrale e all'impegno lavorativo richiesto.

\section{A urine emesse spontaneamente (voided e non sterili)}

- urine da mitto intermedio

- urine da sacchetto

- urine da catetere vescicale a permanenza

- urine da condotto ileale

$B$ urine prelevate asetticamente

- urine da cateterismo estemporaneo

- urine urine da aspirato sovrapubico

- urine da cistoscopia vescicale

- urine da nefrostomia

\section{$C$ urine frazionate}

L'urina è un fluido corporeo normalmente sterile, infatti ad eccezione della parte distale dell'uretra, l'apparato urinario fa parte delle zone sterili dell'organismo. Ma nell'ultimo tratto di circa $1 \mathrm{~cm}$ l'uretra ospita una popolazione essenzialmente batterica che è prevalentemente di provenienza cutanea ed intestinale. Tale popolazione è più numerosa e di maggiore varietà nel sesso femminile, per $\mathrm{i}$ rapporti topografici qui esistenti tra uretra e vestibolo vaginale. Infatti mentre nel maschio troviamo S. epidermidis, Corynebacterium spp, E. coli, Mycobacterium smegmatis; nella donna oltre ai sopracitati microrganismi possiamo trovare altre Enterobacteriaceae, Lattobacilli, Streptococchi aerobi ed anaerobi, Bacteroides, Veillonelle, Candida.

\section{Come ottenere il campione di urine}

Per ottenere un campione idoneo e non contaminato nell'eseguire il prelievo si dovrà tenere conto:

- della "flora" microbica residente dell'uretra anteriore

- della "flora" microbica residente delle zone adiacenti allo sbocco dell'uretra

- dell'azione di lavaggio esercitata dall'urina sulla mucosa vescicale della capacità dell'urina di consentire la crescita e la moltiplicazione batterica

\section{Tempo ottimale per la raccolta del campione}

Ogni volta che è possibile, prima della terapia antibiotica, in caso contrario segnalare al laboratorio la terapia antibatterica in atto. Il tempo di permanenza delle urine in vescica può influenzare notevolmente il numero dei batteri, per cui è importante che il laboratorio stabilisca una precisa regola, un tempo standard che renda omogenei i campioni ai fini dell'interpretazione dei risultati. Per garantire una giusta sosta delle urine in vescica, raccogliere preferibilmente le urine delle prima minzione del mattino (la carica batterica infatti risulta aumentata dopo l'incubazione notturna in vescica) o almeno 3-4 ore dopo l'ultima minzione.

Non sforzare il paziente a bere per emettere le urine; l'introduzione eccessiva di liquidi può diluire le urine e fare abbassare la carica batterica.

\section{Modalità di raccolta}

\section{Urine da mitto intermedio:}

per ottenere un campione idoneo da un paziente in grado di cooperare è sufficiente osservare alcuni accorgimenti; dopo aver accuratamente deterso con sapone e quindi sciacquato ed asciugato l'area periuretrale (estremità del pene, piccole e grandi labbra, vulva) nel maschio si ritrae la cute del prepuzio e dopo aver scartato il primo getto si 
raccoglie direttamente nel recipiente sterile la seconda parte delle urine emesse; il metodo è analogo nella femmina che però dovrà avere l'accortezza di divaricare le grandi labbra.

\section{Urine raccolte con sacchetto sterile adesivo:} tale metodica è utilizzata nei bambini più piccoli o comunque quando sempre nei bambini non è possibile ottenere un campione di urine da mitto intermedio. Per la facilità di contaminazione del campione con materiale fecale o perineale, il ricorso a tale metodica deve essere effettuato con cautela ed eseguito secondo precise istruzioni che sono:

- mantenere i piccoli in posizione eretta

- stimolare opportunamente la minzione del bambino

- detergersi con cura ,sciacquare ed asciugare le mani

- lavare con cura i genitali esterni ed il perineo del piccolo paziente con acqua e sapone, sciacquare ed asciugare

- aprire il sacchetto sterile evitando di toccarne l'interno

- far aderire il sacchetto alla cute perineale

- raccogliere le urine

- richiudere il sacchetto utilizzando l'apposita linguetta adesiva

- porre il sacchetto in posizione verticale in un contenitore a bocca larga

- non travasare le urine dal sacchetto in un altro contenitore.

Nel caso il piccolo paziente abbia difficoltà a urinare, rimuovere il sacchetto ogni 30 minuti e ripetere la procedura di posizionamento fino alla raccolta del campione.

Adulti incontinenti: la raccolta può avvenire esclusivamente tramite catetere.

3. Urine da cateterismo estemporaneo, il suo impiego è da limitare a casi eccezionali, in quanto manovra invasiva di per sé possibile causa di infezione.

- La procedura va eseguita da personale qualificato, rispettando rigorose norme di sterilità.

- Verificare la presenza di globo vescicale.

- Introdurre sterilmente il catetere.

- Lasciare defluire e scartare la prima parte delle urine.

- Raccogliere le urine successive in un contenitore sterile.

4. Urine da catetere a permanenza: la raccolta del campione deve avvenire senza incorrere in possibili contaminazioni del circuito chiuso.

- Clampare il catetere immediatamente a valle del dispositivo di prelievo.
- Disinfettare con un impacco di alcool al 70\% il dispositivo del catetere predisposto per il prelievo.

- Con tecnica sterile aspirare con una siringa la quantità di urina necessaria e dispensarla nell'apposito contenitore sterile.

\section{Tecniche particolari:}

5. Urine da prelievo sovrapubico, il prelievo è utile per accertare un'infezione in soggetti in cui il risultato di precedenti culture sia dubbio e la diagnosi critica.

$\grave{E}$ indicato in pediatria quando non sia possibile la minzione spontanea e nel caso di pazienti con lesioni spinali.

È obbligatorio per diagnosticare un'eventuale infezione da anaerobi.

L'urina è ottenuta asetticamente e direttamente dalla vescica per aspirazione con ago e siringa.

6. Urine raccolte da catetere nefrostomico o da uretere-cutaneo-stomia: questo campione può essere ottenuto da pazienti ai quali sia stata praticata una stomia addominale; la raccolta del campione deve avvenire senza incorrere in possibili contaminazioni nel passaggio dell'urina dal catetere addominale al contenitore di raccolta e contemporaneamente senza determinare contaminazioni esogene del catetere. In ogni caso il campione di urine non può essere prelevato dal sacchetto di raccolta.

7. Urine da cistoscopio o da cateteri ureterali: questi campioni possono essere ottenuti da pazienti sottoposti a cistoscopia, aspirando direttamente dalla vescica e/o dagli ureteri.

8. Urine frazionate o test di Stamey: campioni raccolti per la diagnosi di prostatite batterica cronica. Si prevedono 3 campioni di urine più le secrezioni prostatiche:

- VB1o $1^{\circ}$ campione è il mitto iniziale di circa 5$8 \mathrm{cc}$. che rappresenta l'uretra

- VB2 o $2^{\circ}$ campione è il mitto intermedio che rappresenta la vescica.

- EPS secrezioni prostatiche dopo massaggio

- VB3 o $3^{\circ}$ campione sono le prime urine circa 2$3 \mathrm{cc}$ emesse dopo massaggio prostatico

\section{Modalità di conservazione e trasporto}

Fuori della vescica l'urina non può attendere; la semina immediata permette di evitare false positività.

- Inviare subito in laboratorio e processare entro 2 ore.

- In alternativa conservare in frigo a $4^{\circ}$ per un massimo di 12/24 ore; la crescita batterica sarà inibita, senza che il numero dei germi presenti 
venga alterato in maniera significativa, o raccogliere il campione in appositi contenitori con conservante (acido borico o altri sistemi disponibili in commercio). Seguire le istruzioni del produttore per il rispetto del rapporto campione/conservante.
Norme comportamentali per i pazienti:

- astenersi dai rapporti sessuali nelle 24 ore precedenti l'esame (3-4 giorni per la spermiocoltura).

- aver cessato qualsiasi intervento chemio-antibiotico, sia locale (creme, candelette, ovuli, lavande) che generale, da una settimana (minimo 3 giorni)

\section{Quali campioni per quali esami}

\begin{tabular}{|l|l|}
\hline Campione & Ricerche \\
\hline $\begin{array}{l}\text { Urine raccolte con qualsiasi modalità di prelievo } \\
\text { Inviare immediatamente in laboratorio e processare entro 2h. } \\
\text { Refrigerare a } 4^{\circ} \mathrm{C} \text { per } 12-24 \mathrm{~h} \\
\text { Utilizzare contenitori con conservante (ac. Borico), rispettando il } \\
\text { rapporto urine/conservante; temperatura ambiente fino a 48h }\end{array}$ & $\begin{array}{l}\text { Esame colturale quantitativo per batteri } \\
\text { aerobi, esigenti e miceti }\end{array}$ \\
\hline $\begin{array}{l}\text { Urine da puntura sovrapubica o con cistoscopio } \\
\text { Processare entro } 15 \text { min. o conservare in dispositivo per anaerobi }\end{array}$ & Esame colturale per anaerobi \\
\hline $\begin{array}{l}\text { Primo mitto (almeno } 15 \mathrm{ml} \text { ) della prima minzione del mattino } \\
\text { Consegna immediata o } 24 \mathrm{~h} \text { a } 4^{\circ} \mathrm{C}\end{array}$ & $\begin{array}{l}\text { Es.colturale per Micoplasmi urogenitali, } \\
\text { limitata ai maschi con sintomatologia di } \\
\text { tipo uretrale. } \\
\text { Ricerca Clamydia trachomatis con tecniche } \\
\text { di amplificazione genica }\end{array}$ \\
\hline $\begin{array}{l}\text { Urine raccolte con qualsiasi modalità di prelievo e in qualsiasi } \\
\text { momento della giornata. } \\
\text { Consegna immediata } \circ 24 \mathrm{~h} \text { a } 4^{\circ} \mathrm{C}\end{array}$ & Ricerca Antigene Legionella \\
\hline $\begin{array}{l}\text { La prima urina del mattino } 50-100 \mathrm{cc}(\text { almeno } 40 \text { ) in contenitore sterile } \\
\text { senza conservanti } \\
\text { Alcune linee guida suggeriscono la tecnica del mitto intermedio. } \\
3 \text { campioni in } 3 \text { giorni successivi. } \\
\text { Consegna immediata } \circ 48 \mathrm{~h} \text { a } 4^{\circ} \mathrm{C} \text {, periodo entro il quale è conservata } \\
\text { la vitalità dei Micobatteri }\end{array}$ & Ricerca Micobatteri \\
\hline $\begin{array}{l}\text { Urine raccolte con qualsiasi modalità di prelievo } \\
3 \text { campioni in } 3 \text { giorni successivi } \\
\text { Consegna immediata }\end{array}$ & Ricerca Leptospire \\
\hline
\end{tabular}

- per il tampone endouretrale: astenersi dall'urinare, e per i maschi anche dall'eiaculare, per almeno 3 ore prima del prelievo.

- aspettare almeno 3-4 giorni dalla fine delle mestruazioni non eseguire irrigazioni vaginali nelle 24 ore precedenti l'esame

La scelta dei campioni dipende dalla sede dell'infezione, dal microrganismo sospettato, dalla metodica utilizzata per l'indagine.
Criteri di non accettabilità:

- Campione raccolto con il condom

- Punta e sacca di catetere

- Campioni duplicati nello stesso giorno

- Urine delle $24 \mathrm{~h}$ per ricerca Micobatteri

- Campioni raccolti da $>2 \mathrm{~h}$ e senza evidenza di refrigerazione o conservante.

\section{Campioni dall'apparato genitale, maschile e femminile}

Quando eseguire l'esame microbiologico?

- In presenza di sintomatologia, anche se blanda

- In pazienti giovani al di sotto i 25 anni, visto il più precoce inizio dell'attività sessuale

- Prima di eseguire manovre invasive, quali l'isterosalpingografia o l'inserimento di dispositivi intrauterini

- Se esistono fattori di rischio (partner con infezioni, abitudini alla promiscuità sessuale, pregresse infezioni)

- In gravidanza (minaccia di aborto, parto pretermine, PROM, infezioni connatali)

Come per tutti gli esami microbiologici, l'adeguata preparazione del paziente e l'appropriatezza del prelievo, conservazione e trasporto dei campioni sono presupposti essenziali per assicurare la qualità del risultato.
Materiali prelevati o raccolti dalle vie genitali

\begin{tabular}{ll}
\hline $\begin{array}{l}\text { Pazienti di } \\
\text { sesso femminili }\end{array}$ & $\begin{array}{l}\text { Pazienti di } \\
\text { sesso maschile }\end{array}$ \\
\hline Fluido amniotico & Tampone anale \\
\hline $\begin{array}{l}\text { Aspirato da ghiandola } \\
\text { del Bartholino }\end{array}$ & Tampone balanoprepuziale \\
\hline Prelievo endocervicale & Lesioni dal pene \\
\hline Prelievo endometriale & $\begin{array}{l}\text { Secrezione uretrale } \\
\text { o prelievo endouretrale }\end{array}$ \\
\hline Aspirato da tube di Falloppio & Secrezione prostatica \\
\hline Tampone anale/rettale & Test di Meares-Stamey \\
\hline Raccolta di secrezione & \\
uretrale o prelievo endouretrale & Liquido seminale \\
\hline Prelievo vaginale & \\
\hline Prelievo vulvare & \\
\hline
\end{tabular}

Poiché le infezioni genitali sono localizzate in sede anatomiche normalmente colonizzate da una "flora " microbica residente, per una accurata diagnosi sia nell'uomo che nella donna è necessario distinguere i microrganismi patogeni da quelli normalmente residenti.

Nella donna possono dar luogo a vulviti, cerviciti, endometriti fino a P.I.D. ed uretriti.

I più frequenti responsabili di vulviti sono: miceti lievitiformi (Candida), Herpes simplex Streptococchi.

I più frequenti responsabili di vaginiti sono: Trichomonas vaginale, miceti lievitiformi, Gardnerella vaginalis e Mobiluncus spp. 
I più frequenti responsabili di endocerviciti sono: Chlamydia trachomatis, Neisseria gonorrhoeae.

\begin{tabular}{ll}
\hline $\begin{array}{l}\text { Infezioni genitali } \\
\text { nella donna }\end{array}$ & Microrganismi responsabili \\
\hline Vulviti & Candida Herpes simplex, Streptococchi \\
\hline Vaginiti & $\begin{array}{l}\text { Trichomonas vaginale Candida, } \\
\text { Gardnerella.vaginalis, Mobiluncus spP } \\
\text { aerobi opportunisti }\end{array}$ \\
\hline Endocerviciti & $\begin{array}{l}\text { Chlamydia trachomatis, Neisseria gonor- } \\
\text { rhoeae Micoplasmi urogenitali }\end{array}$ \\
\hline Endometriti e P.I.D. & Anaerobi e come nelle cerviciti \\
\hline Infezioni da IUD & Actinomiceti ed altri anaerobi \\
\hline Uretriti & $\begin{array}{l}\text { Chlamydia trachomatis, Neisseria gonor- } \\
\text { rhoeae Micoplasmi urogenitali, aerobi } \\
\text { opportunisti }\end{array}$ \\
\hline Lesioni genitali & Virus Treponema \\
\hline
\end{tabular}

Nell'uomo le più comuni sedi di infezioni sono l'uretra, la prostata, il solco balano prepuziale. I più frequenti responsabili di uretreti sono Chlamydia trachomatis, Neisseria gonorrhoeae e Micoplasmi. I più frequenti responsabili di prostatiti sono gli stessi microrganismi che causano le infezioni urinarie. Talvolta, come complicazione di una uretrite, può aversi un' infezione prostatica da Chlamydia trachomatis, Neisseria gonorrhoeae e Micoplasmi.I più frequenti responsabili di balanopostiti sono i comuni microrganismi aerobi, anaerobi e miceti lievitiformi che fanno parte della flora microbica residente nella sede interessata e che per particolari condizioni locali si comportano da opportunisti. In entrambi i sessi vanno inoltre presi in considerazione le forme a localizzazione cutanea e/o mucosa che si manifestano con ulcerazioni di vario genere e principalmente dovute nei nostri climi ad infezione luetica

\begin{tabular}{ll}
\hline $\begin{array}{l}\text { Infezioni } \\
\text { genitali nell'uomo }\end{array}$ & Microrganismi responsabili \\
\hline Uretriti & $\begin{array}{l}\text { Chlamydia trachomatis, Neisseria gonor- } \\
\text { rhoeae Micoplasmi urogenitali, aerobi } \\
\text { opportunisti }\end{array}$ \\
\hline Prostatiti & $\begin{array}{l}\text { Microrganismi responsabili di UTI e } \\
\text { Chlamydia trachomatis, Neisseria gonor- } \\
\text { rhoeae Micoplasmi urogenitali }\end{array}$ \\
\hline Balanopostiti & Aerobi, anaerobi e lieviti \\
\hline Lesioni genitali & Virus Treponema \\
\hline
\end{tabular}

Il prelievo deve essere eseguito da personale qualificato ed esperto (ginecologo, ostetrica, urologo, dermatologo, microbiologo medico).

Vanno segnalati, però recenti lavori americani che citano l'opportunità di incoraggiare, tra le adolescenti, il controllo delle malattie sessualmente trasmesse facendo ricorso all'auto-prelievo del tampone, per la ricerca di Chlamydia, Gonococco e
Trichomonas con il metodo del PCR (polymerase chain reaction amplification). Vista l'alta prevalenza di MST in questa popolazione, la scarsa compliance a sottoporsi ai tradizionali controlli ginecologici, l'alta sensibilità diagnostica della metodica, quasi tutti concludono sottolineandone accettabilità e fattibilità, a fronte soprattutto del rischio di molte MST altrimenti non diagnosticate e non trattata.

\section{MATERIALI UTILIZZATI}

La maggior parte dei campioni per la diagnosi di infezioni genitali sono raccolti mediante tampone; non tutti i tipi di tampone in commercio sono ugualmente idonei. Anche se i tamponi in cotone sono accettabili per la ricerca di Trichomonas e la diagnosi di vaginosi batterica ,gli acidi grassi presenti nelle fibre del cotone, e certi componenti del bastoncino di legno e della colla possono invece risultare tossici per la maggior parte dei patogeni del tratto genitale. E opportuno utilizzare tamponi in dacron o rayon su asta di plastica o metallo per tutti i campioni genitali, evitando anche $i$ tamponi in alginato di calcio non idonei per gli isolamenti virali e per tutte quelle metodiche che utilizzano reagenti di estrazione.

Si raccomanda l'uso di spazzolini da citologia (cytobrush) per ottenere materiale con ricca cellularità più idoneo alla ricerca di Chlamydia.

Idealmente i campioni dovrebbero essere trasportati e processati il più presto possibile.

Purtroppo l'assetto organizzativo dei laboratori di microbiologia non sempre lo permette, quindi i tamponi dovrebbero essere inseriti in idonei terreni di trasporto per mantenere la vitalità dei microrganismi, un $\mathrm{pH}$ adeguato, prevenire l'essiccamento e la sovracrescita batterica.

\section{Indagini microbiologiche vaginali}

Prelievo: dal fornice posteriore, previa introduzione di uno speculum bivalve sterile.

Il pH vaginale va misurato per contatto con le pareti laterali prima dell'inserimento dello speculum, utilizzando cartine indicatrici per liquidi ad alto contenuto proteico o pHametri monouso.

Il Fish odor test va eseguito al momento del prelievo o predisporre una provetta con $0.5 \mathrm{ml}$ di soluzione fisiologica in cui stemperare il campione. Allestire i vetrini per l'esame microscopico a fresco e al Gram e i tamponi per le colture.

\section{Indagini microbiologiche cervicali}

Il prelievo deve essere effettuato a livello endocervicale, previa introduzione di speculum bivalve sterile nel modo seguente:

- rimuovere l'eccesso di muco cervicale median- 
te un tampone a secco

- utilizzare altri tamponi per eseguire il prelievo inserendoli nell'endocervice per circa $1 \mathrm{~cm}$. e ruotare più volte. Per la ricerca di C.trachomatis il tampone o meglio il cyto-brush va inserito $1.5 \mathrm{~cm}$. oltre la giunzione squamosa.

La donna gravida può essere sottoposta a prelievo cervicale ad esclusione dell'ultimo trimestre, eseguendolo a livello dell'esocollo. usando esclusivamente il tampone.

\section{Indagini microbiologiche uretrali femminili}

In presenza di emissione spontanea di secrezione raccogliere direttamente il materiale ed allestire strisci e vetrini; in mancanza di secrezioni si effettua il prelievo endouretrale con le seguenti modalità: pulire accuratamente l'orifizio esterno dell'uretra con soluzione fisiologica ed asciugare; inserire gli appositi sottili tamponi per circa $1 \mathrm{~cm}$ nell'uretra, ruotandoli delicatamente. Dopo il prelievo consigliare alla paziente di bere tre o quattro bicchieri d'acqua in modo da stimolare la minzione (il lavaggio dell'uretra con il passaggio delle urine diluite elimina il residuo eventuale bruciore e riduce $\mathrm{il} \mathrm{rischio} \mathrm{di} \mathrm{infezioni)}$

\section{Indagini microbiologiche uretrali maschili}

Indicazione per l'esecuzione del tampone uretrale: a. il tampone uretrale deve essere eseguito in caso di: secrezione purulenta o vischioso-filamentosa dal meato uretrale, con o senza sintomatologia dolorosa;

b.il tampone deve essere eseguito anche nel caso in cui siano stati isolati nel partner microrganismi tipicamente responsabili di MST;

c. il tampone uretrale potrebbe essere utile anche nella ricerca dell'eziologia di una epididimite. Non è invece indicato nella diagnosi delle prostatiti.

d. Altra indicazione all'indagine sono gli screening per l'infertilità.

Ai fini della diagnostica delle infezioni a trasmissione sessuale nell'uomo, l'esame colturale dell'urina primo mitto e/o mitto intermedio può essere utile qualora non sia possibile utilizzare altri materiali per problematiche tecniche o scarsa collaborazione del paziente.

Inoltre può essere di aiuto nella diagnosi delle epididimiti nei pazienti $>35$ anni ed è fortemente raccomandato nelle prostatiti acute, dove la causa più frequente sono patogeni delle vie urinarie.

\section{Tampone uretrale maschile}

In caso di emissione spontanea di secrezione, raccogliere direttamente il materiale e allestire strisci e colture; in mancanza di secrezione eseguire scraping endouretrale con tamponi di piccolo cali- bro dopo detersione con soluzione fisiologica del glande e del meato uretrale esterno; è consigliabile evitare la rotazione del tampone, ma attuare un delicato movimento di va e vieni. Dopo il prelievo, come detto per le pz femminili consigliare al paziente di bere.

Allestire il vetrino per l'esame microscopico direttamente al momento del prelievo e non dal tampone inviato in laboratorio.

Per evitare contaminazioni da parte della normale flora batterica cutanea, il prelievo deve essere preceduto da una accurata pulizia dei genitali esterni e delle mani.

La raccolta del liquido seminale deve avvenire mediante masturbazione e tutto l'eiaculato deve essere raccolto nell'apposito contenitore sterile e consegnato in laboratorio entro 1 ora.

\section{Tampone balanoprepuziale}

Invitare il paziente a scoprire il glande e raccogliere con tampone l'essudato eventualmente presente nella zona del solco balano-prepuziale ed inviarlo in laboratorio immesso nel terreno di trasporto.

Il prelievo da lesioni ulcerate del pene è indicato solo per la ricerca di Treponemi o Herpes simplex direttamente dalle lesioni.

Volendo fare delle considerazioni conclusive sui campioni del tratto genitale, a quanto sopra detto possiamo aggiungere che la coltura per Gonococco dovrebbe essere allestita al momento del prelievo e incubata immediatamente; se ciò non fosse possibile utilizzare tamponi con terreno di trasporto; quello ritenuto più idoneo è l'Amies contenente una soluzione salina tamponata e bilanciata con l'aggiunta di carbone, in quanto capace di inibire gli effetti tossici di ioni e sottoprodotti del metabolismo batterico.

L'Amies è da preferirsi allo Stuart che contenendo glicero-fosfato può facilitare la moltiplicazione delle Enterobacteriaceae.

Se si ritarda la processazione è preferibile conservare i campioni a $4^{\circ} \mathrm{C}$ gradi piuttosto che a temperatura ambiente (per $12 \mathrm{~h}$ e comunque non oltre le 48h). Anche per il Gonococco recenti studi evidenziano una loro migliore sopravvivenza se conservati a $4^{\circ} \mathrm{C}$ gradi e comunque non oltre le 6 12h. Per la ricerca di Haemophilus ducreyi è ammessa solo la semina diretta.

Sono disponibili in commercio specifici terreni di trasporto per Trichomonas, Mycoplasmi, Chlamydia e ricerche virali.

Fluidi e pus dovrebbero essere raccolti per aspirazione e inviati in laboratorio negli appositi sistemi di trasporto per anaerobi contenenti agenti riducenti come il Thioglicolato di sodio; $i$ dispositivi intrauterini per la ricerca di 
Actinomyces vanno inseriti per intero nei contenitori da urinocoltura e trasportati con sistemi per l'anaerobiosi.

Nella donna la tubercolosi genitale comincia con un focus ematogeno nella salpinge, da cui si estende all'endometrio, ovaio, cervice, vagina. La diagnosi è possibile utilizzando alcuni $\mathrm{ml}$ di sangue mestruale, raccolti al $2^{\circ}-3^{\circ}$ giorno di flusso mestruale in provetta con eparina o co materiale da raschiamento endometriale.

Nell'uomo l'infezione tubercolare dal rene può estendersi alla prostata, vescicole seminali, epididimo e testicoli. Materiali utili per la diagnosi sono: urine, liquido seminale, biopsie e aspirati

Considerazioni riassuntive sui campioni dal tratto genitale per esame batteriologico e micobatteriologico

\begin{tabular}{|c|c|c|}
\hline Microrganismo & Campione raccomandato & Conservazione e trasporto \\
\hline N.gonorrhoeae & $\begin{array}{l}\text { Cervicale, anale, uretrale urina } 1^{\circ} \\
\text { mitto nei } O^{\prime} \text { sintomatici }\end{array}$ & $\begin{array}{l}\text { Semina immediata oTampone con } \\
\text { Amies più carbone a } 4^{\circ} \mathrm{C} \text { per } 6-12 \mathrm{~h}\end{array}$ \\
\hline Batteri aerobi & Uretrale, prostatico, cervicale, vaginale & T. con Amies a $4^{\circ} \mathrm{C}$ fino a $48 \mathrm{~h}$ \\
\hline $\begin{array}{l}\text { G. vaginalis per } \\
\text { vaginosi }\end{array}$ & Striscio su vetrino di secrezioni vaginali & $\begin{array}{l}\text { Non fissare conservazione e trasporto } \\
\text { non critici }\end{array}$ \\
\hline Batteri anaerobi & Fluidi aspirati pus & $\begin{array}{l}\text { Semina immediata o sistemi di } \\
\text { trasporto per anaerobi, T. ambiente } \\
\text { fino a } 48 \mathrm{~h}\end{array}$ \\
\hline Actinomiceti & IUD & Semina immediata \\
\hline C.trachomatis & Uretrale, cervicale, urina $I^{\circ}$ mitto & $\begin{array}{l}\text { Vetrini per immunofluorescanza o } \\
\text { specifici sistemi di trasporto per } \\
\text { amplificazione genica }\end{array}$ \\
\hline $\begin{array}{l}\text { Micoplasmi } \\
\text { urogenitali }\end{array}$ & $\begin{array}{l}\text { Uretrale, cervicale, sperma urina } 1^{\circ} \\
\text { mitto e liquido gastrico nel neonato }\end{array}$ & $\begin{array}{l}\text { Specifici terreni di trasporto } \\
\text { T.ambiente fino a } 8 \mathrm{~h} \mathrm{a} 4^{\circ} \mathrm{C} \text { fino a } 36 \mathrm{~h}\end{array}$ \\
\hline T. pallidum & $\begin{array}{l}\text { Vetrino da lesioni cutanee per es. in } \\
\text { campo oscuro o immunofluorescenza }\end{array}$ & Conservazione e trasporto non critici \\
\hline Micobatteri & $\begin{array}{l}\text { Nella donna: alcuni } \mathrm{ml} \text { di sangue } \\
\text { mestruale, raccolti al } 2^{\circ}-3^{\circ} \text { giorno } \\
\text { di flusso mestruale in provetta con } \\
\text { eparina o materiale da } \\
\text { raschiamento endometriale } \\
\text { - Nell'uomo: urina, liquido seminale, } \\
\text { biopsie e aspirati }\end{array}$ & Processazione immediata \\
\hline
\end{tabular}

\section{PARTE \\ Giorgio Mucignat}

\section{EMOCOLTURA}

La fase preanalitica è un elemento del percorso diagnostico microbiologico di fondamentale importanza per l'efficacia e la significatività dell'esame. Ciò vale ovviamente ed in particolare anche per l'esame emocoltura.

Esso dipende da alcune attenzioni da porre nell'eseguirlo. Importante è il

\section{MOMENTO DEL PRELIEVO}

che deve avvenire secondo alcune regole:

- prima della terapia antibiotica

- prima della nuova somministrazione di antibiotico

- 2 ore prima del rialzo febbrile (quando prevedibile)
- durante la puntata febbrile (o nei $30 \mathrm{~min}$. seguenti)

Nel caso la terapia antibiotica sia già in atto, per vari motivi, risulta comunque possibile eseguire un esame emocolturale grazie a due ordini di accorgimenti tecnici che sono disponibili per il microbiologo:

1. la diluizione del campione di sangue $(1: 5-1: 10)$ in grado di diluire dello stesso fattore l'antibiotico,

2.1'utilizzo di chelanti (carbone o resine) in grado di sottrarre molecole antibiotiche dal sangue.

\section{ACCURATEZZA DEL PRELIEVO}

Dipende da precise modalità di prelievo che devono essere messe in atto per evitare la contaminazione esogena del campione con conseguente difficoltà interpretativa del risultato. Sappiamo infatti che la flora epiteliale (Es. Staphylococcus epidermidis) può contaminare il campione e renderne difficoltosa l'interpretazione della positività)

\section{CARATTERISTICHE DEL PRELIEVO}

Il prelievo deve essere eseguito utilizzando due flaconi, uno per flora aerobia ed uno per quella anaerobia, che vengono inoculati uno di seguito all'altro.

\section{PER ADULTI:}

1 - 3 prelievi a distanza di almeno 20-30 minuti (batteriemie intermittenti) o più.

2 - flaconi di coltura per aerobi e anaerobi (meglio con dispositivi per annullare l'effetto degli antibiotici o diluizione 1:10)

3 - volume totale di sangue prelevato $20-30 \mathrm{ml}$ (ciò garantisce il 99\% degli isolamenti possibili)

\section{PEDIATRICO:}

Nel caso di bambini, in riferimento al minore volume sanguigno ed alla scarsissima frequenza di anaerobi tra le cause di setticemia, si utilizza un solo flacone per prelievo per aerobi nel seguente modo:

$1-1-3 \mathrm{ml}$ di sangue

2 - 2-3 volte nella giornata

3 - flaconi appositi con terreno di coltura per aerobi

Nel caso di sospetto coinvolgimento di anaerobi il clinico dovrebbe informare il microbiologo che provvederà in merito fornendo il corretto flacone. 


\section{CONSERVAZIONE DEL CAMPIONE}

Gli strumenti automatici per emocoltura attualmente in uso prevedono il mantenimento del campione trasportato a temperatura ambiente.

L'attenzione deve esser posta nell'inserimento dei campioni il prima possibile nel sistema analitico, anche favorendo l'inserimento da parte di personale sanitario che esegue il trasporto (nei momenti in cui non sia disponibili il personale tecnico di laboratorio.

\section{EMOCOLTURE ADDIZIONALI}

Nel caso di prelievi ripetuti, essi vanno eseguiti in giornate successive alla prima, e quindi dopo 2448 ore (in genere non più di tre volte) in:

- pazienti che non rispondono alla terapia

- nuovo episodio febbrile

- quando il primo prelievo non si sia positivizzato É qui evidente la forte necessità di una tempestiva comunicazione dell'eventuale positività dell'emocoltura al clinico.

\section{MICRORGANISMI CHE NECESSITANO MODALITA' COLTURALI DIVERSE}

Il sospetto clinico di setticemia da microrganismi particolari indurrà il microbiologo a modificare, rispetto, agli standard, le procedure analitiche. I casi più frequenti riguardano i seguenti batteri:

- Miceti (incubare almeno 2 settimane)

- Brucella spp. (incubare 4 settimane)

- Leptospira (terreni appositi)

- Legionella spp. (incubare 2 settimane e subcoltivare negli appositi terreni)

- Micobatteri: > terreni appositi per i diversi sistemi analitici

$>$ prelevare da 5 a $10 \mathrm{ml}$ in provetta con SPS o eparina o ISOLATOR

Per quanto riguarda l'Isolator, esso rappresenta una soluzione tecnica volta a concentrare gli eventuali batteri presenti nel sangue ed a seminarli direttamente negli appositi terreni di coltura. Ciò comporta una manipolazione aperta del sangue, con un aumento conseguente del rischio da parte dell'operatore, perciò va utilizzato con raziocinio ed estrema attenzione alle procedure di sicurezza.

\section{MATERIALE VIE RESPIRATORIE}

Nel caso dell'apparato respiratorio dividiamo, per comodità, le procedure per BASSE VIE ed ALTE VIE RESPIRATORIE.
BASSE VIE

\section{ESAMI DI UTILITÀ CLINICA}

Se si sospetta un'infezione in questo distretto di possono analizzare diversi materiali, tra cui:

1. emocolture

2. es. colturale liquido pleurico

3. materiali ottenuti con tecniche invasive broncoaspirato, BAL, aspirato transcricoideo, biopsia polmonare

4. espettorato

5. tecniche sierologiche su siero (per indicazioni epidemiologiche)

Il campione forse più comune ma sicuramente non il più semplice da ottenere ed esaminare è l'ESPETTORATO

Esso deve essere:

1. raccolto prima della terapia antibiotica

2. contenitore sterile con tappo a vite

3. al mattino dopo gargarismo con acqua distillata oppure induzione con soluzione salina (3-10\%)

Deve essere inviato entro 15 minuti in laboratorio per mantenere i rapporti originari di flora batterica. Campioni che pervengono in laboratorio dopo 2 ore non dovrebbero essere esaminati (falsi "positivi" o "negativi") se non mantenuti a $4^{\circ} \mathrm{C}$.

Questo campione andrà valutato con appropriate tecniche atte a stabilire se vi sia una eccessiva contaminazione salivare e nel caso verrà sucessivamente scartato, in quanto non rappresentativo delle basse vie respiratorie. Nonostante la difficoltà di ottenere un buon campione bisogna ricordare che un solo campione ben raccolto e ben conservato è sufficiente per una corretta diagnostica.

Migliori campioni si possono ottenere con le cosiddette.

\section{TECNICHE INVASIVE}

Esse sono :

- aspirato endotracheale (secrezioni che ristagnano in trachea)

- aspirato da tracheostomia (controllo di colonizzazione)

- lavaggio bronchiale

- lavaggio broncoalveolare (BAL) per le infezioni interstiziali

- spazzolato endobronchiale

- biopsia transbronchiale

- biopsia polmonare e pleurica

- liquido pleurico 
Questi materiali vanno raccolti e trasportati nel modo seguente:

- raccolto in contenitore sterile

- trasporto sollecito (15 min)

- conservazione 2 ore a $+4^{\circ} \mathrm{C}$

- per i materiali normalmente sterili è possibile, quando il laboratorio sia chiuso, inoculare il campione in flaconi di coltura (quelli normalmente usati per emocolture) per aerobi e anaerobi.

\section{RICERCA MICOBATTERI}

Sono previste le stesse modalità di prelievo, conservazione e trasporto per tutti i materiali, come per altri batteri. In particolare è utile ottenere per l'espettorato, 3 campioni in mattine successive. Nel caso di positività dell'esame diretto del primo campione è superfluo inviare nuovi campioni. Ricordiamo a questo proposito che l'esame diretto, nel caso sia positivo, deve essere refertato entro 24 ore dal ricevimento.

Per quanto riguarda quest'ultimo tipo di campioni viene riportata di seguito una tabella riassuntiva della tipologia e delle modalità di trasporto dei campioni.

Tabella I.guida al trasporto campioni

\begin{tabular}{|c|c|}
\hline TIPO CAMPIONE & METODO DI TRASPORTO \\
\hline BIOPSIA POLMONARE & $\begin{array}{l}\text { VASETTO STERILE CON TAPPO A VITE } \\
\text { (se la campionatura e piccola usare vasetto } \\
\text { sterile con I-2 ml di salina) }\end{array}$ \\
\hline $\begin{array}{l}\text { ESPETTORATO } \\
\text { (spontaneo e indotto) }\end{array}$ & VASETTO STERILE CON TAPPO A VITE \\
\hline LAVAGGIO & TRAPPOLA PER SPUTO ○ VASETTO \\
\hline $\begin{array}{l}\text { BRONCOALVEOLARE o } \\
\text { BRONCHIALE }\end{array}$ & $\begin{array}{l}\text { STERILE CON TAPPO A VITE 。 } \\
\text { PROVETTA STERILE }\end{array}$ \\
\hline $\begin{array}{l}\text { BIOPSIA } \\
\text { TRANSBRONCHIALE }\end{array}$ & $\begin{array}{l}\text { VASETTO STERILE CON TAPPO A VITE } \\
\text { con } 1-2 \mathrm{~m} \text { I di salina }\end{array}$ \\
\hline "BRUSH" BRONCHIALE & $\begin{array}{l}\text { VASETTO STERILE CON TAPPO A VITE } \\
\text { con } 1-2 \mathrm{~m} \text { I di salina }\end{array}$ \\
\hline $\begin{array}{l}\text { ASPIRATO } \\
\text { TRANSTRACHEALE 。 } \\
\text { POLMONARE }\end{array}$ & $\begin{array}{l}\text { SISTEMA DI TRASPORTO PER ANAEROBI } \\
\text { ○ VASETTO STERILE CON TAPPO A VITE } \\
\text { o PROVETTA STERILE }\end{array}$ \\
\hline
\end{tabular}

\section{ALTE VIE RESPIRATORIE}

Per quanto riguarda questo distretto possono essere inviati i seguenti materiali:

- essudato faringeo (evitare contaminazioni orali)

- essudato nasale (non per diagnosi di sinusite)

- aspirato seni paranasali

- essudato auricolare (orecchio esterno e interno)

- essudato orale (ricerca miceti e fusospirilli per Angina di Vincent)

\section{MATERIALE PER ESAME COLTURALE}

Normalmente questi materiali vengono raccolti per mezzo di tamponi con terreno di trasporto. Essi rendono possibile un tempo di mantenimento, tra il prelievo e la semina al massimo 48 ore a temperatura ambiente.

Tuttavia letteratura recente ha messo in evidenza la variabilità, da ditta a ditta della qualità del mezzo di trasporto oltre che la variabilità di comportamento tra $i$ vari batteri. Sarebbe perciò utile utilizzarli considerando un periodo di "sicurezza" di 6-12 ore.

Viene riportata di seguito una tabella (tabella 2) riassuntiva della tipologia e delle modalità di trasporto di questo gruppo di campioni.

Tabella 2. guida al trasporto campioni

\begin{tabular}{|l|l|}
\hline \multicolumn{1}{|c|}{ TIPO CAMPIONE } & \multicolumn{1}{|c|}{ METODO DI TRASPORTO } \\
\hline TAMPONE FARINGEO & $\begin{array}{l}\text { SISTEMA DI TRASPORTO TAMPONE } \\
\text { CON TERRENO SOLIDO }\end{array}$ \\
\hline TAMPONE NASALE & $\begin{array}{l}\text { SISTEMA DI TRASPORTO TAMPONE } \\
\text { CON TERRENO SOLIDO }\end{array}$ \\
\hline FLUIDO DA TIMPANOCENTESI & $\begin{array}{l}\text { SISTEMA DI TRASPORTO PER } \\
\text { ANAEROBI O SIRINGA SENZ'AGO } \\
\text { TAPPATA }\end{array}$ \\
\hline ASPIRATO DA SENI NASALI & $\begin{array}{l}\text { SISTEMA DI TRASPORTO PER } \\
\text { ANAEROBI O SIRINGA SENZ'AGO } \\
\text { TAPPATA }\end{array}$ \\
\hline $\begin{array}{l}\text { SUZIONE NASOFARINGEA E } \\
\text { LAVAGGIO NASALE }\end{array}$ & $\begin{array}{l}\text { SISTEMA DI TRASPORTO PER } \\
\text { ANAEROBI O SIRINGA SENZ'AGO } \\
\text { TAPPATA }\end{array}$ \\
\hline
\end{tabular}

\section{ESSUDATI VARI}

Questo è un materiale di varia provenienza caratterizzato da una discreta omogeneità. Ho ritenuto utile riportare la definizione scolastica che è la seguente:

"Materiale che è filtrato attraverso le pareti di cellule viventi, e si accumula in tessuti adiacenti". (Voc. della Lingua italiana. Treccani)

Questo materiale può provenire dai seguenti distretti:

\section{LESIONI APERTE:}

piaghe, ulcere, ustioni, ferite, ferite chirurgiche: Modalità di prelievo: prima del prelievo detersione con soluzione fisiologica sterile (attenzione non bisogna usare soluzione disinfettante)

\section{LESIONI CHIUSE:}

vescicole, pustole, foruncoli:

Modalità di prelievo: disinfettare la zona prima del prelievo

ferite profonde, ascessi, aspirati tessuti molli:

Modalità di prelievo: aspirare asetticamente con siringa ed inviare al laboratorio dopo averla tappata biopsie transcutanee, frammenti ossei:

Modalità di prelievo: umidificare con fisiologica sterile (qualche goccia, massimo un millilitro)

Viene riportata di seguito una tabella (Tabella 3) riassuntiva della tipologia e delle modalità di trasporto di questo gruppo di campioni 
Tabella 3. guida al trasporto campioni

\begin{tabular}{|l|l|l|}
\hline \multicolumn{2}{|c|}{ TIPO CAMPIONE } & \multicolumn{1}{c|}{ METODO DI TRASPORTO } \\
\hline $\begin{array}{l}\text { TESSUTI SUBCUTANEI E } \\
\text { ULCERE O NODULI E } \\
\text { FERITE SUPERFICIALI }\end{array}$ & ESSUDATO & SIRINGA SENZ'AGO TAPPATA \\
\cline { 2 - 3 } & BIOPSIA & $\begin{array}{l}\text { VASETTO STERILE CON TAPPO A VITE } \\
\text { (se la campionatura e piccola usare vasetto } \\
\text { sterile con I-2 ml di salina) }\end{array}$ \\
\hline USTIONI & VASETTO STERILE CON TAPPO A VITE \\
\hline $\begin{array}{l}\text { MATERIALE DA LESIONE SUPERFICIALE } \\
\text { FUNGINEA }\end{array}$ & VASETTO STERILE CON TAPPO A VITE \\
\hline FERITE & IN SEDE & SISTEMA DI TRASPORTO PER ANAEROBI \\
\cline { 2 - 3 } & $\begin{array}{l}\text { PROFONDE O } \\
\text { ASCESSI }\end{array}$ & VASETTO STERILE CON TAPPO A VITE \\
\hline ASPIRATI DA TESSUTI MOLLI & SIRINGA SENZ'AGO TAPPATA \\
\hline OSSO & $\begin{array}{l}\text { VASETTO STERILE CON TAPPO A } \\
\text { VITE(se la campionatura e piccola usare } \\
\text { vasetto sterile con I-2 ml di salina) }\end{array}$ \\
\hline
\end{tabular}

\section{LIQUIDI CAVITARI (normalmente sterili)}

Anche in questo caso è importante porre attenzione ad alcuni passaggi, che sono:

- aspirazione in maniera asettica

- contenitore sterile con tappo a vite

- trasportare subito in laboratorio oppure inoculare in flaconi per emocolture (per aerobi e anaerobi) da tenere a temperatura ambiente.

L'inoculazione nel flaconi per emocoltura è sicuramente una modalità che comporta una certa "comodità"; essa è atta a superare alcune difficoltà organizzative del laboratorio, tuttavia, a mio parere, impedisce di attuare un esame diretto del campione che è sempre di alto valore per il clinico.

Sarebbe perciò opportuno cercar di avere sempre anche un'aliquota di campione per l'esame diretto.

Di seguito, nella tabella (tabella 4) riassuntiva si riporta sia la tipologia che le modalità di trasporto di questo gruppo di campioni.

Tabella 4. guida al trasporto campioni

\begin{tabular}{|c|c|}
\hline TIPO CAMPIONE & METODO DI TRASPORTO \\
\hline SANGUE & $\begin{array}{l}\text { BOTTIGLIE PER EMOCOLTURA ० } \\
\text { PROVETTA TIPO VACUTAINER CON SPS } \\
\text { (se sospetta Neisseria senza SPS) }\end{array}$ \\
\hline LIQUOR & PROVETTA STERILE CON TAPPO A VITE \\
\hline $\begin{array}{l}\text { ASCESSO CEREBRALE o BIOPSIA } \\
\text { CEREBRALE }\end{array}$ & $\begin{array}{l}\text { SISTEMA DI TRASPORTO PER ANAEROBI } \\
\text { (se la campionatura e piccola usare vasetto } \\
\text { sterile con } \quad \mathrm{l}-2 \mathrm{ml} \text { di salina) }\end{array}$ \\
\hline $\begin{array}{l}\text { "SCRAPING" CONGIUNTIVALE E } \\
\text { CORNEALE }\end{array}$ & $\begin{array}{l}\text { INOCULARE I TERRENI E SPATOLARE I } \\
\text { VETRINI DIRETTAMENTE }\end{array}$ \\
\hline FLUIDO INTRAOCULARE & $\begin{array}{l}\text { INOCULARE I TERRENI E SPATOLARE I } \\
\text { VETRINI DIRETTAMENTE O SISTEMA DI } \\
\text { TRASPORTO ANAEROBI o SIRINGA } \\
\text { SENZ'AGO TAPPATA E SENZA ARIA } \\
\text { INTERNA }\end{array}$ \\
\hline $\begin{array}{l}\text { LIQUIDO PLEURICO, PERITONEALE, } \\
\text { ASCITICO, SINOVIALE }\end{array}$ & $\begin{array}{l}\text { VASETTO STERILE CON TAPPO A VITE o } \\
\text { SIRINGA SENZ'AGO TAPPATA o SISTEMA } \\
\text { DI TRASPORTO ANAEROBI o sist. per } \\
\text { emocoltura }\end{array}$ \\
\hline
\end{tabular}

\section{CANNULE ENDOVASCOLARI}

Per questo tipo di campione è utile:

- prelievo in maniera asettica

- tagliare sterilmente la punta $(5 \mathrm{~cm})$

- in contenitore sterile

- trasportare subito in laboratorio oppure aggiungere qualche goccia di fisiologica sterile e tenere $\mathrm{a}+4^{\circ} \mathrm{C}$ per non più di 24 ore

\section{$\underline{\text { RICERCA MICOBATTERI }}$}

- Non usare $\mathrm{i}$ tamponi di trasporto in quanto $\mathrm{i}$ Micobatteri tendono a rimanere aderenti ai filamenti mentre si "scarica" il tampone, ciò a causa dell'elevata idrofobicità della loro parete.

- umidificare i materiali solidi con fisiologica sterile

- mantenere il materiale prelevato a $+4^{\circ} \mathrm{C}$ fino a 24 ore

\section{RECEPIMENTO ED ACCETTAZIONE DEI CAMPIONI}

Quando arrivano in laboratorio i campioni vanno correttamente vagliati e vanno messe in atto precise procedure di cui si da di seguito elencazione.

\section{ARRIVO CAMPIONI}

- documentare il tempo di ricevimento

- verificare presenza di identificazione

- assegnare un numero di laboratorio

- esaminare visualmente il campione

- verificare l'appropriatezza del campione rispetto all'indagine richiesta

- determinare appropriatezza del contenitore:

- terreno di trasporto

- rotture o fuoriuscite

Si possono così evidenziare situazioni di non conformità agli standard previsti. Nel caso, a seconda delle situazioni si procede come indicato di seguito:

\section{CAMPIONI NON IDENTIFICATI O MALE IDENTIFICATI}

QUESTI CAMPIONI NON SONO ACCETTABILI!

Tuttavia non si deve rigettare il campione sino a quando si sappia che è possibile ottenerne un altro (soprattutto in riferimento al tipo di campione) e deve essere documentata la ragione dell'inaccettabilità.

\section{CAMPIONI DUPLICATI}

NON DOVREBBERO ESSERE LAVORATI!

(escludendo emocolture, Liquidi da cavità chiuse, 
tessuti).

Se sono campioni raccolti in tempi ravvicinati si possono lavorare come unico campione.

Notificare al richiedente "campione duplicato: esame non eseguito. Vedi referto $N^{\circ} \ldots . .$.

Non lavorare più di tre campioni in giorni consecutivi.

\section{CONTENITORI ROTTI}

IL CAMPIONE NON È ACCETTABILE; se è pesantemente imbrattato in esterno dal campione stesso (verificare se è possibile ottenerne un altro prima di gettarlo)

Se il contenitore è rotto e non è possibile ottenerne un altro, verificare se sia possibile lavorarlo senza rischi per l'operatore.

\section{CAMPIONI CONTAMINATI}

Non accettare campioni contaminati da altri prodotti biologici (es: urine con feci ecc.)

Attivare procedura di richiesta di un nuovo campione

\section{CAMPIONI INACETTABILI}

Sono inaccettabili i seguenti campioni:

- Non accettare campioni di saliva invece di escreato.

- Punta di catetere di Foley per coltura.

- Campioni da piaga di decubito solo se prelevati chirurgicamente o in modo da escludere la contaminazione di superficie.

- Non accettare urine di 24 ore per colturale

\section{CAMPIONI IN RITARDO}

Il ritardo di un campione presuppone la conoscenza dell'ora di prelievo. Questa viene raramente riportata sul foglio di richiesta o sul campione stesso. Questo rimane quindi un fatto critico ed importante per la valutazione da farsi all'arrivo del campione. $\mathrm{E}$ inoltre importante che il laboratorio semini il campione al più presto ed eventualmente attui misure di mantenimento refrigerato (non emocolture e liquor) dei campioni. Va assicurata la precedenza ai campioni per ricerca anaerobi.

Idealmente comunque quasi tutti $i$ campioni dovrebbero non superare le due ore di trasporto.

\section{CONTENITORI PER TRASPORTO}

\section{USARE SOLO CONTENITORI STERILI!!}

Il contenitore tappo vite per urine può essere usato per altri liquidi corporei , escreati e tessuti il tappo del contenitore non deve creare aerosol quando viene rimosso.

Non usare capsule Petri perché non ermetiche.
Tutti i contenitori dovrebbero essere chiusi in busta di plastica con la richiesta attaccata all'esterno.

I campioni raccolti con tampone a secco sono inaccettabili (eccezione fatta per la richiesta di SBA Ag).

\section{BIBLIOGRAFIA}

1. Henry D. Hisenberg Clinical Microbiology Procedures Handbook 1992 Vol 1 Specimen collection and Transport.

2. Van Hom, Toth C, Wegienek J. Viability of aerobic microrganisms in four swab systems. ASM 98 th General Meeting. Atlanta, Georgia, May 1998.

3. Lauer BA, Masters HB. Toxic effect of calcium alginate on Neisseria gonorrhoeae J Clin Microbiol 1988 Jan; 26(1): 54-6.

4. Mandler F, Sfondrini D. Evaluation of survival of bacteria on dry swabs and transport system. Ann Sclavo 1977 Jul-Aug; 19(4); 537-45.

5. NCCLS. Document M40-P Quality Control of Microbiological Transport Systems; Proposed Standard (ISBN 1 -56238-465-1) USA 2002.

6. Perry JL, Matthews JS. Compliance of Two Popular Swab Transport Systems with Performance Standards Detailed by the new NCCLS Proposed Standard, M40-P. ASM $103^{\text {rd }}$ General Meeting, Washington DC. May 2003.

7. Center for Disease Control and Prevention. Recommendations for the Collection of Laboratory Specimens associates with outbreaks of gastroenterics. MMWR 1990, 39.

8. AMCLI - CoSMic. Proposta di linee guida per la diagnosi microbiologica dei micobatteri. Microbiologia Medica 1999; 14(4).

9. Wilson ML. General principles of specimen collection and transport. Clin Infect Dis 1996 May; 22: 766-77.

10. NCCLS Urinalysis and Collection, Transportation and Preservation of Urine Specimens: Approved guideline - Second Edition, GP16-A2, 2001; 21(19).

11. PHLS standard operating procedure. B.SOP 28 INVESTIGATION OF GENITAL TRACT AND ASSOCIATED SPECIMENS Issue N. 3 Issue date 20.09.02 Issued by: Technical Services, PHLS HQ.

12. Health Protection Agency BSOP 30. Investigation of faces specimens for bacterial pathogens Issue $n^{\circ} 3.1$ Issue date: 02.06 .03

13. Health Protection Agency BSOP 30-Investigation of specimens for mycobacterium species. Issue $\mathrm{N}^{\circ}$ 4.1.Issue date 02.06.03 BSOP41 Health Protection Agency Investigation of urine species Issue $n^{\circ}$ 3.1.Issue date 02.06.03 Issued by: Standards Unit, Evoluations and Standards Laboratory.

14. TML/MSH Microbioloy Department; Policy and Procedure Manual; Section: Enteric Culture Manual; issued 27 march 2000.

15. TML/MSH Microbioloy Department; Policy and Procedure Manual; Section: Urine Culture Manual; issued 2 February 2000.

16. TML/MSH Microbioloy Department; Policy and Procedure Manual; Section: Genital tract Culture Manual; issued 8 march 2000. 